\title{
ON THE ALGEBRA OF SIEGEL MODULAR FORMS OF GENUS 2
}

\author{
E. B. VINBERG
}

\begin{abstract}
Using the methods of our 2010 paper, we recover the old result of J. Igusa, saying that the algebra of even Siegel modular forms of genus 2 is freely generated by forms of weights $4,6,10,12$. We also determine the structure of the algebra of all Siegel modular forms of genus 2 and, in particular, interpret the supplementary generator of odd weight as the Jacobian of the generators of even weights.
\end{abstract}

\section{INTRODUCTION}

In 1962, J. Igusa [5] (see also [6], 7], 10]) proved that the algebra of even Siegel modular forms of genus 2 was freely generated by forms of weights $4,6,10,12$. Up to recently, this was essentially the only example of when the structure of the algebra of automorphic forms on a symmetric domain of dimension greater than 2 was completely determined.

In 2010, the author 14 proved that some algebras of automorphic forms on symmetric domains of type IV of dimensions 4,5,6,7 were free, and found the weights of their generators. The proof was based on the interpretation of the corresponding arithmetic quotients of symmetric domains of type IV as the moduli spaces for some classes of quartic surfaces. In dimension 3, the symmetric domain of type IV is nothing other than the Siegel upper half-space $\mathcal{S}_{2}$ of genus 2. Making use of this fact, we recover the result of Igusa by the method of [14. This proof has the advantage (or disadvantage?) that it does not involve any "explicit" formulas for automorphic forms such as Eisenstein series.

The freeness of the algebra of even Siegel modular forms of genus 2 implies that the action of the Siegel modular group $\Gamma_{2}$ on $\mathcal{S}_{2}$ is generated by reflections. Since for $g>2$ the Siegel upper half-space $\mathcal{S}_{g}$ of genus $g$ does not admit reflections, the algebra of even Siegel modular forms of genus $g>2$ cannot be free.

We also prove that the algebra of all Siegel modular forms of genus 2 is generated by forms of weights $4,6,10,12,35$, where the square of the odd generator is expressed as a polynomial in the even generators. (The explicit form of this polynomial can be found in [7.) In a sense, the odd generator is the Jacobian of the even ones (see Section 3). It vanishes exactly on the mirrors of the group $\Gamma_{2}$.

It is really surprising that the same family of quartic surfaces was independently considered in 2]. We are grateful to I. Dolgachev, who drew our attention to that publication.

This work was partially supported by the RFBR grant No. 11-01-00289. It was completed during the author's stay at Bielefeld University, in June-August of 2012, and published as a preprint there [15. The author thanks this university for its hospitality.

2010 Mathematics Subject Classification. Primary 05A10, 11A07, 11C20, 11R04, 11S15.

Key words and phrases. Symmetric domain, automorphic form, reflection group, moduli space, quartic surface, K3 surface, period map, categorical quotient. 


\section{GRoups AND BUNDLES}

The Siegel upper half-space $\mathcal{S}_{g}$ of genus $g$ is (a model of) the Hermitian symmetric space $\mathrm{Sp}_{2 g}(\mathbb{R}) / \mathrm{U}_{g}$, the symmetric domain of type III. The Siegel modular group of genus $g$ is the group $\Gamma_{g}=\mathrm{Sp}_{2 g}(\mathbb{Z})$. It is known that it is a maximal discrete subgroup in $\mathrm{Sp}_{2 g}(\mathbb{R})$; see, e.g., [1]. (There must be a more adequate reference, but we did not find such.)

A Siegel modular form of genus $g$ and weight $k$ is a $\Gamma_{g}$-invariant holomorphic section of the $k$-th power of a certain $\operatorname{Sp}_{2 g}(\mathbb{R})$-equivariant line bundle over $\mathcal{S}_{g}$. This bundle is uniquely determined by the condition that its $(g+1)$-th power is the canonical bundle.

Another series of symmetric domains is formed by the symmetric domains of type IV, which are the Hermitian symmetric spaces

$$
\mathcal{D}_{n}=\mathrm{SO}_{2, n}^{+} /\left(\mathrm{SO}_{2} \times \mathrm{SO}_{n}\right)=\mathrm{O}_{2, n}^{+} /\left(\mathrm{SO}_{2} \times \mathrm{O}_{n}\right),
$$

where $\mathrm{O}_{2, n}^{+}$denotes the subgroup of index 2 of $\mathrm{O}_{2, n}$ consisting of the elements whose spinor norm is equal to the determinant, and $\mathrm{SO}_{2, n}^{+}=\mathrm{O}_{2, n}^{+} \cap \mathrm{SO}_{2, n}$.

For $n \geq 3$, an automorphic form of weight $k$ on $\mathcal{D}_{n}$ with respect to a lattice $\Gamma \subset \mathrm{O}_{2, n}^{+}$ is a $\Gamma$-invariant holomorphic section of the $k$-th power of a certain $\mathrm{O}_{2, n}^{+}$-equivariant line bundle over $\mathcal{D}_{n}$, whose $n$-th power is the canonical bundle. If

$$
\pi: \mathcal{L}_{n} \rightarrow \mathcal{D}_{n}
$$

is the corresponding $\mathbb{C}^{*}$-bundle (obtained by deleting the zero section), then automorphic forms of weight $k$ can be viewed as $\Gamma$-invariant holomorphic functions on $\mathcal{L}_{n}$ that are homogeneous of degree $-k$ on each fiber.

In this work, we exploit the isomorphism $\mathcal{S}_{2} \simeq \mathcal{D}_{3}$, which gives rise to some isomorphisms of the algebras of automorphic forms. Below we describe this isomorphism in detail.

Considering the natural representation of $\mathrm{SL}_{4}(\mathbb{R})$ in $\wedge^{2} \mathbb{R}^{4}$, one obtains a homomorphism of $\mathrm{SL}_{4}(\mathbb{R})$ to $\mathrm{SO}_{3,3}$, whose kernel is $\{ \pm E\}$, and the image is the subgroup $\mathrm{SO}_{3,3}^{+}$ of index 2 of $\mathrm{SO}_{3,3}$ consisting of the elements of spinor norm 1. Restricting this homomorphism to $\mathrm{Sp}_{4}(\mathbb{R})$ gives rise to an epimorphism

$$
\rho: \mathrm{Sp}_{4}(\mathbb{R}) \rightarrow \mathrm{SO}_{2,3}^{+} .
$$

The kernel of $\rho$ is contained in $\mathrm{U}_{2}$, so $\rho$ induces an isomorphism between the symmetric spaces $\mathcal{S}_{2}=\mathrm{Sp}_{4} / \mathrm{U}_{2}$ and $\mathcal{D}_{3}=\mathrm{SO}_{2,3}^{+} /\left(\mathrm{SO}_{2} \times \mathrm{SO}_{3}\right)$. Note that the equivariant line bundles on these two spaces involved in the definitions of automorphic forms are taken to one another by this isomorphism, since they both are the cubic roots of the canonical bundle.

More precisely, if $\left\{e_{1}, e_{2}, e_{3}, e_{4}\right\}$ is the standard basis of $\mathbb{R}^{4}$, then the scalar product in $\wedge^{2} \mathbb{R}^{4}$ is given by

$$
\left(e_{1} \wedge e_{2}, e_{3} \wedge e_{4}\right)=\left(e_{1} \wedge e_{3}, e_{4} \wedge e_{2}\right)=\left(e_{1} \wedge e_{4}, e_{2} \wedge e_{3}\right)=1,
$$

with all the other products of the basis bivectors $e_{i} \wedge e_{j}$ being equal to 0 . The group $\mathrm{Sp}_{4}(\mathbb{R})$ fixes the bivector $e_{1} \wedge e_{2}+e_{3} \wedge e_{4}$ and leaves invariant its orthogonal complement spanned by

$$
f_{0}=e_{1} \wedge e_{2}-e_{3} \wedge e_{4}, f_{1}=e_{1} \wedge e_{3}, f_{2}=e_{4} \wedge e_{2}, f_{3}=e_{1} \wedge e_{4}, f_{4}=e_{2} \wedge e_{3} .
$$

In the basis $\left\{f_{0}, f_{1}, f_{2}, f_{3}, f_{4}\right\}$ the scalar square in the orthogonal complement is given by the quadratic form 


$$
q=-2 x_{0}^{2}+2 x_{1} x_{2}+2 x_{3} x_{4}
$$

Set

$$
\begin{gathered}
\Gamma=\mathrm{O}^{+}(q, \mathbb{Z}) \subset \mathrm{O}_{2,3}^{+}, \\
\Gamma_{0}=\mathrm{SO}^{+}(q, \mathbb{Z}) \subset \mathrm{SO}_{2,3}^{+} .
\end{gathered}
$$

Clearly, the group $\Gamma_{2}$ leaves invariant the lattice generated by $f_{0}, f_{1}, f_{2}, f_{3}, f_{4}$, so $\rho\left(\Gamma_{2}\right) \subset \Gamma_{0}$. But since $\Gamma_{2}$ is a maximal discrete subgroup in $\operatorname{Sp}_{4}(\mathbb{R})$, this inclusion cannot be strict; that is,

$$
\rho\left(\Gamma_{2}\right)=\Gamma_{0}
$$

This yields an isomorphism between the (graded) algebra of Siegel modular forms of genus 2 and the (graded) algebra of automorphic forms on $\mathcal{D}_{3}$ with respect to the group $\Gamma_{0}$.

Moreover, we have

$$
\Gamma=\Gamma_{0} \times\{ \pm E\}
$$

which means that the algebra of automorphic forms on $\mathcal{D}_{3}$ with respect to $\Gamma$ is just the even part of the algebra of automorphic forms with respect to $\Gamma_{0}$ and, hence, is isomorphic to the algebra of even Siegel modular forms of genus 2.

\section{LATTICES AND ROOTS}

Following the idea of [14, we are going to find a class of multipolarized $K 3$ surfaces parametrized by the points of $\mathcal{D}_{3} / \Gamma$. To this end, we will introduce and investigate some quadratic lattices which are intended to play the roles of the lattices of algebraic and transcendental cycles of our $K 3$ surfaces.

We use the following notation: $A$;

$[A]$ : the quadratic lattice, the scalar product of which is given by a (symmetric) matrix

$[c] L$ : the quadratic lattice obtained from a quadratic lattice $L$ by multiplying all scalar products by $c$;

$L \oplus M$ : the (orthogonal) direct sum of quadratic lattices $L$ and $M$;

$k L$ : the direct sum of $k$ copies of a quadratic lattice $L$;

$L^{*} \subset L \otimes \mathbb{Q}$ : the dual lattice of a lattice $L$.

We denote by $I_{k, l}$ the group $\mathbb{Z}^{k+l}$ equipped with the scalar product

$$
(x, y)=x_{1} y_{1}+\cdots+x_{k} y_{k}-x_{k+1} y_{k+1}-\cdots-x_{k+l} y_{k+l} .
$$

If $k, l>0$, it is the only (up to isomorphism) odd unimodular quadratic lattice of signature $(k, l)$.

For $k \equiv l(\bmod 8)$, we define the quadratic lattice

$$
J_{k, l}=\left\{x \in I_{k, l} \otimes \mathbb{Q}: 2 x_{i} \in \mathbb{Z}, x_{i}-x_{j} \in \mathbb{Z}(i, j=1, \ldots, k+l), \sum_{i} x_{i} \in 2 \mathbb{Z}\right\} .
$$

If $k, l>0$, it is the only even unimodular quadratic lattice of signature $(k, l)$. In particular, the lattice

$$
U=\left[\begin{array}{ll}
0 & 1 \\
1 & 0
\end{array}\right]
$$

is isomorphic to $J_{1,1}$, and, more generally, the lattice $k U$ is isomorphic to $J_{k, k}$.

Recall that, for a $K 3$ surface $X$, the middle homology lattice $H_{2}(X, \mathbb{Z})$ is isomorphic to $J_{3,19}$. 
The following two even quadratic lattices will be of special interest to us:

$$
\begin{aligned}
& S_{0}=[2] \oplus J_{0,16}, \\
& T_{0}=[-2] \oplus 2 U .
\end{aligned}
$$

The scalar square in $T_{0}$ is given by the quadratic form $q$ as defined by (11), so the group $\mathrm{O}^{+}\left(T_{0}\right)$ coincides with the group $\Gamma$ introduced in the previous section.

It is easy to see that the lattice $[2] \oplus[-2]$ admits only one non-trivial extension (of index 2) which is isomorphic to $U$. It follows that the lattice $S_{0} \oplus T_{0}$ admits only one non-trivial extension (also of index 2) which is isomorphic to $3 U \oplus J_{0,16} \simeq J_{3,19}$. Thereby the lattice $S_{0} \oplus T_{0}$ turns out to be embedded into $J_{3,19}$ as a primitive sublattice of index 2. This will allow us to consider the class of $K 3$ surfaces having $S_{0}$ and $T_{0}$ as the lattices of algebraic and transcendental cycles, respectively.

Clearly, any automorphism of the lattice $S_{0} \oplus T_{0}$ (uniquely) extends to an automorphism of $J_{3,19}$. In particular, any automorphism of $T_{0}$ extends to an automorphism of $J_{3,19}$ acting trivially on $S_{0}$.

A primitive vector $\alpha$ of a quadratic lattice $L$ is called a root or, more precisely, a $k$-root if $(\alpha, \alpha)=-k<0$ and the reflection

$$
R_{\alpha}: x \mapsto x+\frac{2(\alpha, x)}{k} \alpha
$$

leaves $L$ invariant, or, equivalently, $k \mid 2(\alpha, x)$ for every $x \in L$. This automatically holds if $k=1$ or 2 . If the lattice $L$ is unimodular, there are no other possibilities, but in general there may be $k$-roots with $k \neq 1,2$. The reflection $R_{\alpha}$ defined by a $k$-root $\alpha$ is called a $k$-reflection.

In this paper we, however, will deal only with 2-roots and the corresponding reflections, and, moreover, one can prove that the lattices we are going to consider have only 2-roots. For this reason, in order to avoid unnecessary complications, we will use the term "root" for 2-roots only.

A quadratic lattice $L$ is called hyperbolic if its signature is $(1, m)$. In this case, the group $\mathrm{O}^{+}(L)$ is a cofinite discrete group of motions of the $m$-dimensional Lobachevky space $H^{m}$ modelled as a connected component of the hyperboloid $(x, x)=1$ in the Minkowski space $L \otimes \mathbb{R}$. The linear reflections $R_{\alpha}$ of the Minkowski space defined by vectors $\alpha$ with negative squares correspond to reflections in hyperplanes of the space $H^{m}$. The group $W(L) \subset \mathrm{O}^{+}(L)$ generated by all 2-reflections of the lattice $L$ is called its Weyl group.

Let $P(L)$ be a fundamental polyhedron of $W(L)$ in $H^{m}$ (which is defined up to the action of $W(L))$, and let $A(L)$ be the corresponding closed polyhedral convex cone in the Minkowski space $L \otimes \mathbb{R}$ so that $P(L)=A(L) \cap H^{m}$.

The cone $A(L)$ is bounded by the mirrors of some reflections of $W(L)$. The corresponding roots looking outside $A(L)$ are called the simple roots of $L$. They can be effectively found by means of an algorithm described in 13 .

We will use the model of the lattice $S_{0}$ described as follows. Consider a real quadratic vector space with a fixed basis $\left\{e_{0}, e_{1}, \ldots, e_{16}\right\}$ and the scalar product

$$
(x, y)=2 x_{0} y_{0}-x_{1} y_{1}-\cdots-x_{16} y_{16} .
$$

The lattice $S_{0}$ will be considered embedded into this space as

$$
S_{0}=\left\{\sum_{i=0}^{16} x_{i} e_{i}: x_{0} \in \mathbb{Z}, 2 x_{i} \in \mathbb{Z}, x_{i}-x_{j} \in \mathbb{Z}(i, j=1, \ldots, 16), \sum_{i=1}^{16} x_{i} \in 2 \mathbb{Z}\right\} .
$$


One can find the simple roots of $S_{0}$ by means of the algorithm of [13], taking $e_{0} / \sqrt{2}$ for the base point. They turn to be the following vectors:

$$
\begin{aligned}
& \alpha_{i}=-e_{i}+e_{i+1} \quad(i=1, \ldots, 15), \quad \alpha_{16}=-e_{15}-e_{16}, \\
& \alpha_{17}=e_{0}+2 e_{1}, \\
& \alpha_{18}=e_{0}+e_{1}+e_{2}+e_{3}+e_{4}, \\
& \alpha_{19}=e_{0}+\frac{1}{2} \sum_{i=1}^{16} e_{i} .
\end{aligned}
$$

Their Coxeter diagram (with their numbers indicated) is shown on Figure 1. One can observe that it does not contain Lanner subdiagrams, and every connected parabolic subdiagram is included into a parabolic subdiagram of rank 15 . This ensures that the convex polyhedron

$$
P=P\left(S_{0}\right)=\left\{x \in H^{16}:\left(\alpha_{i}, x\right) \geq 0 \quad \text { for } \quad i=1, \ldots, 16\right\}
$$

has finite volume and, hence, is a fundamental polyhedron of the group $W=W\left(S_{0}\right)$; see [13, Proposition 1 and Subsection 2.3].

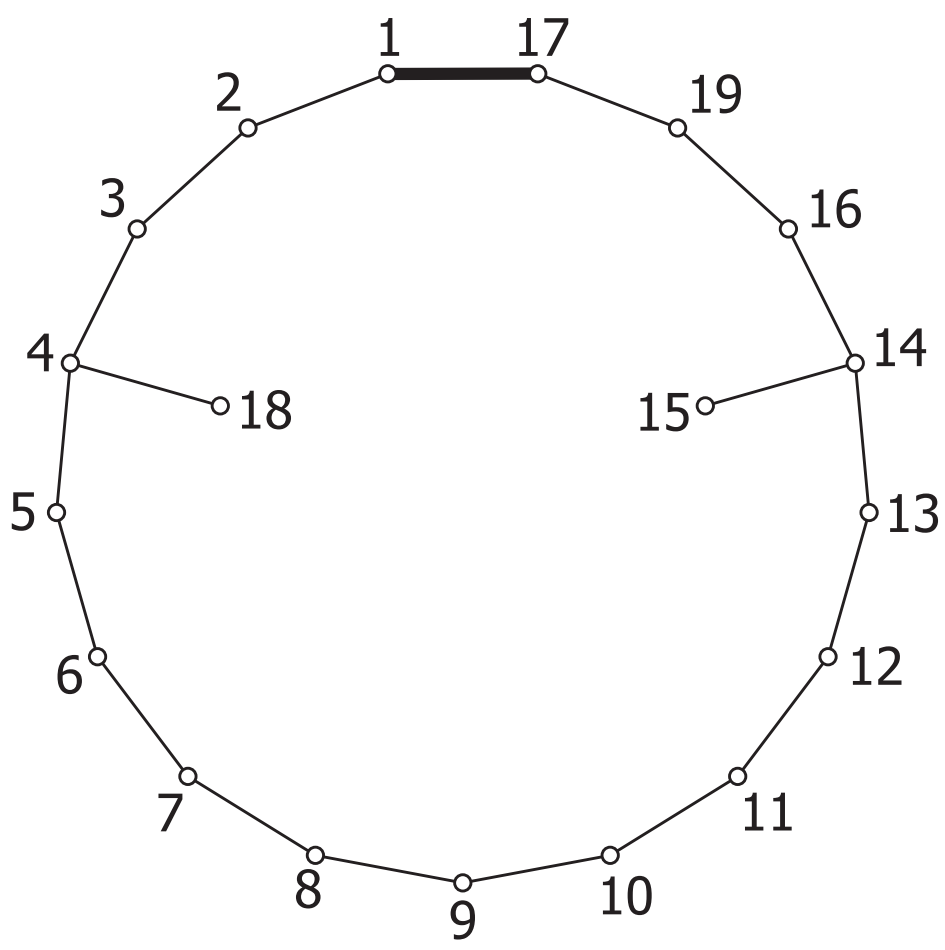

FiguRe 1

Remark 1. This diagram first appeared in [9, Subsection 4.5] (see also [4]).

\section{Multipolarized $K 3$ surfaces}

For a $K 3$ surface $X$, we denote by $S(X)$ and $T(X)$ the lattices of algebraic and transcendental cycles of $X$, respectively. Recall that $S(X)$ is a primitive hyperbolic sublattice of the lattice $H_{2}(X, \mathbb{Z}) \simeq J_{3,19}$ and that $T(X)$ is its orthogonal complement. We denote by $A(X)$ the closure of the convex cone in the Minkowski space $S(X) \otimes \mathbb{R}$ 
generated by the classes of ample divisors. If the signature of $S(X)$ is $(1, m)$, then $P(X)=A(X) \cap H^{m}$ is a fundamental polyhedron of the group $W(X)$ generated by all 2-reflections contained in $\mathrm{O}^{+}(S(X))$. (See, e.g., [3, Proposition 5.10].)

Let $h_{0} \in J_{3,19}$ be a primitive vector with $\left(h_{0}, h_{0}\right)=d>0$, and let $S_{0} \subset J_{3,19}$ be a primitive hyperbolic sublattice of signature $(1, m)$ containing $h_{0}$. Let $T_{0}$ be the orthogonal complement of $S_{0}$ in $J_{3,19}$. It is a primitive sublattice of signature $(2, n)$, where $n=19-m$. A multipolarization of type $\left(h_{0}, S_{0}\right)$ of a $K 3$ surface $X$ is a vector $h \in S(X) \cap A(X)$ and a hyperbolic sublattice $S \subset S(X)$ containing $h$ such that there exists an isomorphism $\varphi: H_{2}(X, \mathbb{Z}) \rightarrow J_{3,19}$ taking $h$ to $h_{0}$ and $S$ to $S_{0}$.

A non-zero regular differential 2 -form $\omega$ on $X$ (defined up to a scalar multiplication) by the Poincaré duality can be considered as an element of $H_{2}(X, \mathbb{C})=H_{2}(X, \mathbb{Z}) \otimes \mathbb{C}$. Then

$$
\varphi(\omega) \in \tilde{\mathcal{L}}_{n}=:\left\{z \in T_{0} \otimes \mathbb{C}:(z, z)=0,(z, \bar{z})>0\right\} .
$$

We will require in addition that $\varphi(\omega) \in \mathcal{L}_{n}$, where $\mathcal{L}_{n}$ is a fixed connected component of the cone $\tilde{\mathcal{L}}_{n}$ (which has two complex conjugate connected components). Then $\varphi$ is defined up to a left multiplication by a transformation of the group $\mathrm{O}^{+}\left(J_{3,19}\right)$, leaving $h_{0}$ and $S_{0}$ (or, equivalently, $h_{0}$ and $\left.T_{0}\right)$ invariant. Denote by $\mathrm{O}^{+}\left(T_{0}, h_{0}\right)$ the group formed by the restrictions of such transformations to $T_{0}$. It is a subgroup of finite index in $\mathrm{O}^{+}\left(T_{0}\right)$.

A quadruple $(X, h, S, \omega)$, where $(X, h, S)$ is a multipolarized $K 3$ surface of type $\left(h_{0}, S_{0}\right)$ and $\omega$ is a non-zero regular differential 2-form on $X$, will be called normed multipolarized $K 3$ surfaces of type $\left(h_{0}, S_{0}\right)$. Via the map $(X, h, S, \omega) \mapsto \varphi(\omega)$ the normed multipolarized $K 3$ surfaces of type $\left(h_{0}, S_{0}\right)$ are parameterized by the points of the variety $\mathcal{L}_{n} / \mathrm{O}^{+}\left(T_{0}, h_{0}\right)$.

The projectivization of $\mathcal{L}_{n}$ is a model of the $n$-dimensional symmetric domain $\mathcal{D}_{n}$ of type IV. Thus, the multipolarized $K 3$ surfaces of type $\left(h_{0}, S_{0}\right)$ are parameterized by the points of the variety $\mathcal{D}_{n} / \mathrm{O}^{+}\left(T_{0}, h_{0}\right)$.

The variety $\mathcal{L}_{n} / \mathrm{O}^{+}\left(T_{0}, h_{0}\right)$ is naturally identified with a Zariski open subset in the spectrum of the algebra $A\left(\mathcal{D}_{n}, \mathrm{O}^{+}\left(T_{0}, h_{0}\right)\right)$ of automorphic forms on $\mathcal{D}_{n}$ with respect to the group $\mathrm{O}^{+}\left(T_{0}, h_{0}\right)$, the boundary being of dimension $\leq 2$. The variety $\mathcal{D}_{n} / \mathrm{O}^{+}\left(T_{0}, h_{0}\right)$ is then identified with a Zariski open subset in the projective spectrum of the algebra $A\left(\mathcal{D}_{n}, \mathrm{O}^{+}\left(T_{0}, h_{0}\right)\right)$, the boundary being of dimension $\leq 1$.

The typical situation for a multipolarized $K 3$ surface $X$ is that $S(X)=S$. If this holds, the multipolarization is called irrational; otherwise it is called rational.

In this paper, we will consider the multipolarized $K 3$ surfaces of type $\left(h_{0}, S_{0}\right)$, where $S_{0} \subset J_{3,19}$ is the sublattice introduced in the previous section and

$$
h_{0}=4 e_{0}+4 e_{1}+e_{2}+\cdots+e_{13} \in S_{0}
$$

in the basis used in (6). The non-zero scalar products of $h_{0}$ with simple roots are indicated in Figure 2.

As we noted above, any automorphism of $T_{0}$ extends to an automorphism of $J_{3,19}$ acting trivially on $S_{0}$, so

$$
\mathrm{O}^{+}\left(T_{0}, h_{0}\right)=\mathrm{O}^{+}\left(T_{0}\right)=\Gamma,
$$

where $\Gamma$ is the group introduced in Section 2.

Moreover, in the considered case, $\left(h_{0}, h_{0}\right)=4$. This means that if $(X, h, S)$ is a multipolarized $K 3$ surface of type $\left(h_{0}, S_{0}\right)$, then the linear system $|h|$ defines a morphism

$$
\varphi_{h}: X \rightarrow \mathbb{C} P^{3} \text {. }
$$

It is known that $\varphi_{h}$ is a birational morphism onto its image if and only if the following condition holds:

(*) There are no isotropic vectors $u \in S(X) \cap A(X)$ with $(h, u)=1$ or 2. 


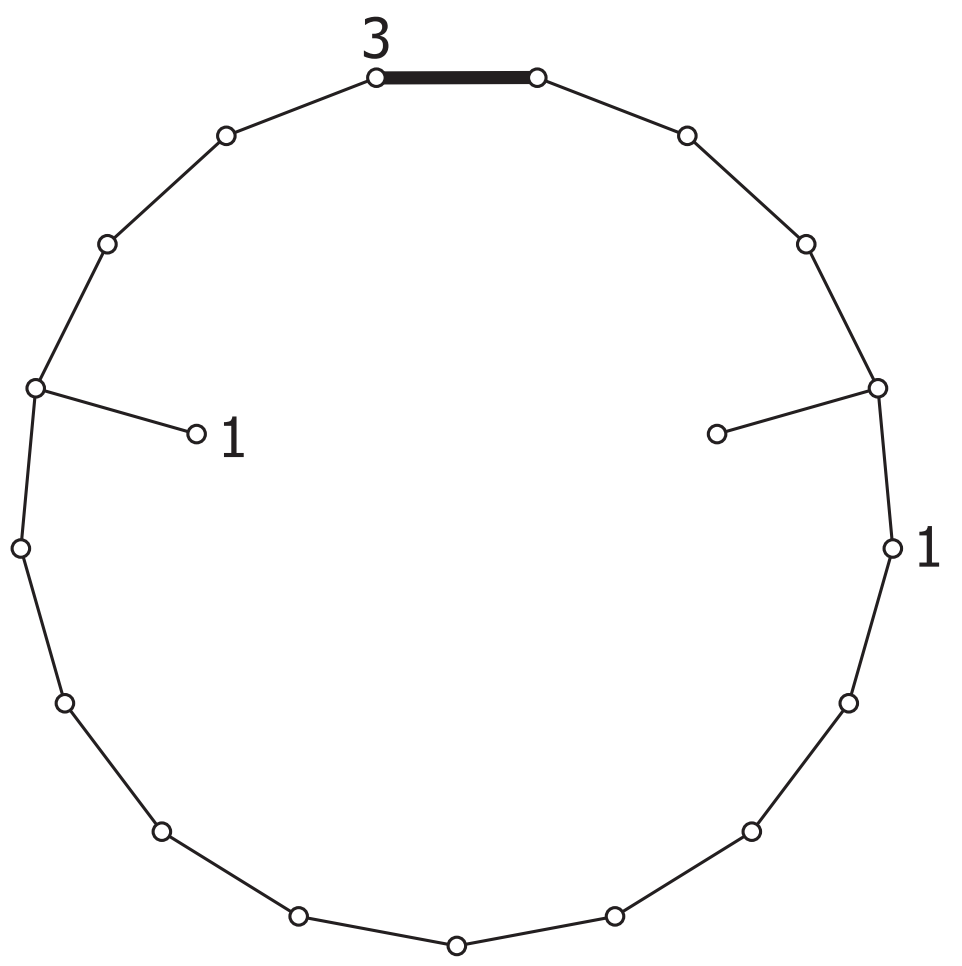

Figure 2

Under this condition, $Y=\varphi_{h}(X)$ is a quartic surface with at most simple singularities. The morphism $\varphi_{h}$ retracts into singular points, the smooth rational curves on $X$ whose classes are orthogonal to $h$, and it is an isomorphism beyond them.

As follows from the next proposition, property $(*)$ holds for all multipolarized $K 3$ surfaces considered in this paper.

Proposition 1. There are no isotropic vectors $u \in J_{3,19}$ with $\left(h_{0}, u\right)=1$ or 2 such that the sublattice generated by $S_{0}$ and $u$ is hyperbolic.

Proof. First, we will show that there are no such vectors $u$ in the very lattice $S_{0}$. According to [14, Lemma 1], it suffices to test the isotropic vectors from $S_{0} \cap A\left(S_{0}\right)$. Using the algorithm described in [14, Subsection 1.6], we see that the cone $A\left(S_{0}\right)$ has exactly three isotropic edges, corresponding to a parabolic subdiagram of type $\tilde{D}_{14}+\tilde{A}_{1}$ and two parabolic subdiagrams of type $\tilde{E}_{8}+\tilde{E}_{7}$, and if $u_{1}, u_{2}, u_{3}$ are the primitive vectors of these edges, then $\left(h_{0}, u_{1}\right)=\left(h_{0}, u_{2}\right)=3,\left(h_{0}, u_{3}\right)=5$.

Suppose now that there is an isotropic vector $u \in J_{3,19} \backslash S_{0}$ satisfying the conditions of the proposition. We have $u=u^{\prime}+u^{\prime \prime}$ with $u^{\prime} \in S_{0}^{*}, u^{\prime \prime} \in T_{0}^{*}$. By our assumption the sublattice generated by $S_{0}$ and $u$ is hyperbolic. This means that $\left(u^{\prime \prime}, u^{\prime \prime}\right)<0$, whence $\left(u^{\prime}, u^{\prime}\right)>0$. Moreover, since $2 S_{0}^{*} \subset S_{0}$, we have $\left(2 u^{\prime}, 2 u^{\prime}\right) \in 2 \mathbb{Z}$. Taking into account that the Gram determinant of $h_{0}$ and $u^{\prime}$ cannot be positive, we obtain that $\left(h_{0}, u^{\prime}\right)=2$ and $\left(u^{\prime}, u^{\prime}\right)=1$ or $\frac{1}{2}$.

In the first case the Gram determinant equals 0 , which means that $u^{\prime}=\frac{1}{2} h_{0}$. But it is easy to see that $\frac{1}{2} h_{0} \notin S_{0}^{*}$, a contradiction.

In the second case we have $\left(2 u^{\prime}, 2 u^{\prime}\right)=2$, so $2 u^{\prime}=h_{0}+\alpha$, where

$$
\left(h_{0}, \alpha\right)=0, \quad(\alpha, \alpha)=-2,
$$


i.e. $\alpha$ is a root orthogonal to $h_{0}$. Every such root is obtained from a simple root orthogonal to $h_{0}$ by applying some element of $W\left(S_{0}\right)$ fixing $h_{0}$. For any simple root $\alpha$ orthogonal to $h_{0}$, there is another simple root $\beta$ orthogonal to $h_{0}$ such that $(\alpha, \beta)=1$ (see Figure 2), whence $h_{0}+\alpha \notin 2 S_{0}^{*}$. But then this is also true for any root $\alpha$ orthogonal to $h_{0}$, a contradiction.

\section{Quartics}

We will use the following notation from [14:

$Q$ : the $S L_{4}$-module of quartic forms in 4 variables;

$T$ : the maximal torus of $S L_{4}$ consisting of diagonal matrices;

$Y(F) \subset \mathbb{C} P^{3}(F \in Q, F \neq 0)$ : the surface defined in homogeneous coordinates by the equation $F=0$;

$Q^{\circ} \subset Q$ : the Zariski open subset consisting of the forms $F \in Q$ such that the surface $Y(F)$ is irreducible and has at most simple singularities;

$X(F)\left(F \in Q^{\circ}\right)$ : the $K 3$ surface obtained by the desingularization of $Y(F)$;

$\omega(F)$ : the regular differential 2-form on $X(F)$ canonically defined as in 14, Subsection 2.2] (recall that $\omega(t F)=t^{-1} \omega(F)$ for $t \in \mathbb{C}^{*}$ ).

Now let $(X, h, S, \omega)$ be a normed multipolarized $K 3$ surface of type $\left(h_{0}, S_{0}\right)$, where $S_{0} \subset J_{3,19}$ and $h_{0} \in S_{0}$ are defined by (6) and (7). Proposition 1 assures that the linear system $|h|$ defines a birational morphism

$$
\varphi_{h}: X \rightarrow Y \subset \mathbb{C} P^{3},
$$

where $Y=Y(F)$ is an irreducible quartic surface with at most simple singularities. The form $F$ will be supposed normalized by the condition $\omega(F)=\omega$.

Proposition 2. Let $Y=Y(F)\left(F \in Q^{\circ}\right)$, and let $X=X(F)$ be the corresponding $K 3$ surface. Assume that $X$ admits an irrational multipolarization $(h, S)$ of type $\left(h_{0}, S_{0}\right)$, where $h \in H_{2}(X, \mathbb{Z})$ is the class of (the pullback of) a plane section of $Y(F)$. Then the form $\mathrm{F}$ is $S L_{4}$-equivalent to a form

$$
F_{0}=A x_{0}^{2} x_{2} x_{3}+B x_{1}^{3} x_{3}+C x_{2}^{4}+x_{2} x_{3} f_{2}\left(x_{1}, x_{2}, x_{3}\right),
$$

where $f_{2}$ is a quadratic form not containing $x_{1}^{2}$ and $x_{3}^{2}$, and $A, B, C \neq 0$. The form $F_{0}$ is uniquely defined up to the action of $T$.

Proof. By our assumption $S(X)=S$. Considering the scalar products of $h$ with the simple roots $\alpha_{1}, \ldots, \alpha_{19}$ of $S$ (see Figure 2), we see that $\varphi_{h}$ retracts to points all the smooth rational curves on $X$ but $\alpha_{1}, \alpha_{13}, \alpha_{18}$, and maps $\alpha_{13}$ and $\alpha_{18}$ to some lines on $Y$, say, $l_{1}$ and $l_{2}$. (We denote smooth rational curves on $X$ as the corresponding simple roots.) The quartic $Y$ has two singular points, say $o$ and $p$, of types $A_{11}$ and $A_{5}$, both lying on $l_{1}$, and $o$ also lying on $l_{2}$.

The non-connected parabolic subdiagrams of the Coxeter diagram yield linear dependences between simple roots (see [14, Subsection 1.6]). In our case, we obtain

$$
\begin{gathered}
\alpha_{1}+\alpha_{17}=\alpha_{18}+\alpha_{3}+2\left(\alpha_{4}+\cdots+\alpha_{14}\right)+\alpha_{15}+\alpha_{16}, \\
2 \alpha_{2}+4 \alpha_{3}+6 \alpha_{4}+5 \alpha_{5}+4 \alpha_{6}+3 \alpha_{7}+2 \alpha_{8}+\alpha_{9}+3 \alpha_{18} \\
=\alpha_{11}+2 \alpha_{12}+3 \alpha_{13}+4 \alpha_{14}+3 \alpha_{16}+2 \alpha_{19}+\alpha_{17}+2 \alpha_{15}, \\
\alpha_{1}+2 \alpha_{2}+3 \alpha_{3}+4 \alpha_{4}+3 \alpha_{5}+2 \alpha_{6}+\alpha_{7}+2 \alpha_{18} \\
=\alpha_{9}+2 \alpha_{10}+3 \alpha_{11}+4 \alpha_{12}+5 \alpha_{13}+6 \alpha_{14}+4 \alpha_{16}+2 \alpha_{10}+3 \alpha_{15} .
\end{gathered}
$$

The vector $h$ can be expressed as a linear combination of simple roots as follows:

(12) $h=3 \alpha_{2}+6 \alpha_{3}+9 \alpha_{4}+8 \alpha_{5}+7 \alpha_{6}+6 \alpha_{7}+5 \alpha_{8}+4 \alpha_{9}+3 \alpha_{10}+2 \alpha_{11}+\alpha_{12}+4 \alpha_{18}$.

Other expressions can be found using (9)-(11). 
Denote by $\bar{S}$ the subgroup of $S$ generated by the simple roots orthogonal to $h$. It follows from (12) and (91)-(11) that

$$
h \equiv 4 \alpha_{18} \equiv 3 \alpha_{13}+\alpha_{18} \quad(\bmod \bar{S}) .
$$

This means that there is a plane $P_{1} \subset \mathbb{C} P^{3}$ such that

$$
P_{1} \cap Y=4 l_{2}
$$

and a plane $P_{2} \subset \mathbb{C} P^{3}$ such that

$$
P_{2} \cap Y=3 l_{1}+l_{2} .
$$

The configuration $\left(o, p, l_{1}, l_{2}, P_{1}, P_{2}\right)$ is canonically associated with $Y$. Applying a suitable projective transformation, we may (and will) assume that

$$
\begin{aligned}
& o=(1: 0: 0: 0), \quad p=(0: 0: 0: 1), \\
& l_{1}: x_{1}=x_{2}=0, \quad l_{2}: x_{2}=x_{3}=0, \\
& P_{1}: x_{3}=0, \quad P_{2}: x_{2}=0 .
\end{aligned}
$$

(See Figure 3).

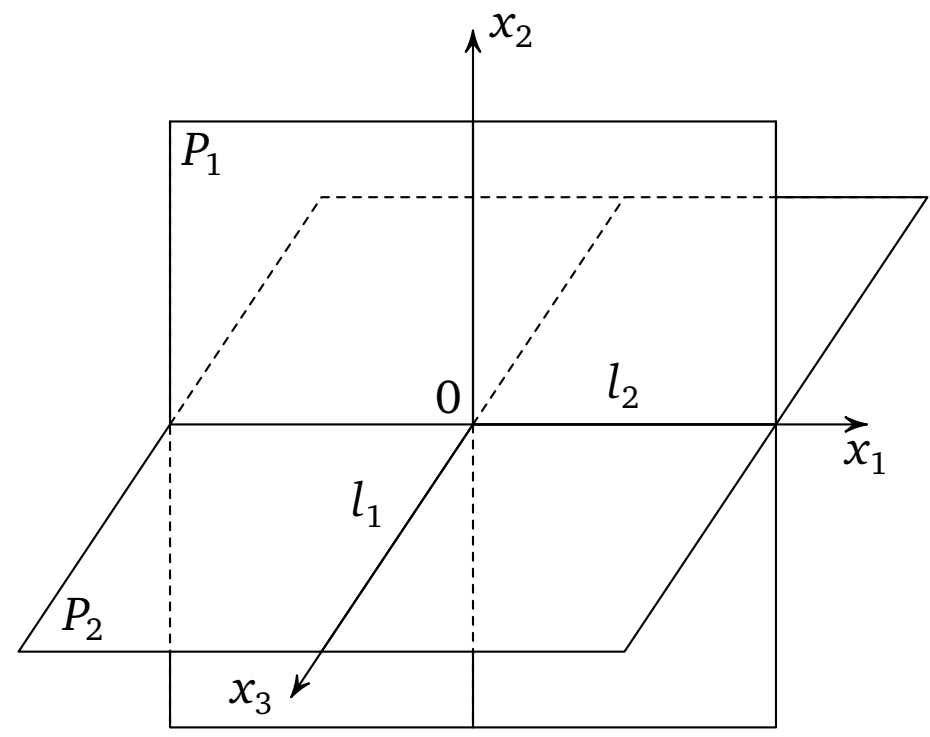

FiguRe 3

Under these assumptions, conditions (13) and (14) mean that

$$
F=B x_{1}^{3} x_{3}+C x_{2}^{4}+x_{2} x_{3} g_{2}\left(x_{0}, x_{1}, x_{2}, x_{3}\right),
$$

where $g_{2}$ is a quadratic form not containing $x_{3}^{2}$. (The latter follows from the assumption that $p=(0: 0: 0: 1)$ is a singular point of $Y$.) Clearly, $B, C \neq 0$, since otherwise $F$ would be divisible by $x_{2}$ or $x_{3}$.

There is an automorphism of the lattice $J_{3,19}$ acting trivially on $S_{0}$ (in particular, fixing $h_{0}$ ) and as -1 on $T_{0}$. According to [11, this means that there is an involution $\delta$ of $X$ leaving invariant all smooth rational curves on $X$ and multiplying $\omega$ by -1 . Moreover, $\delta$ is realized as a projective involution of $Y$ (which we will denote with the same letter), leaving invariant all the elements of the configuration $\left(o, p, l_{1}, l_{2}, P_{1}, P_{2}\right)$.

Again applying a suitable projective transformation, we may assume that $\delta$ is defined by a diagonal linear operator $D$ with \pm 1 on the diagonal. Since $\delta$ multiplies $\omega$ by -1 , 
we have two possibilities: either $D F=F$ and $\operatorname{det} D=-1$, or $D F=-F$ and $\operatorname{det} D=1$. However, the second possibility is not realized because of the non-zero term $C x_{2}^{4}$ of $F$. Hence, $\operatorname{det} D=-1$, and, replacing $D$ with $-D$ if needed, we may assume that $D$ just multiplies one coordinate by -1 . Considering the condition $D F=F$, we see from (16) that the only possibility is that

$$
D=\operatorname{diag}(-1,1,1,1),
$$

which means that $x_{0}$ occurs with even exponents in all non-zero terms of $F$, i.e.,

$$
F=A x_{0}^{2} x_{2} x_{3}+B x_{1}^{3} x_{3}+C x_{2}^{4}+x_{2} x_{3} f_{2}\left(x_{1}, x_{2}, x_{3}\right),
$$

where $f_{2}$ is a quadratic form not containing $x_{3}^{2}$. Here $A \neq 0$, since otherwise $o=(1$ : $0: 0: 0)$ is not a simple singularity of $Y$ (the second differential of the local equation vanishes at $o$ ).

Adding to $x_{1}$ a suitable multiple of $x_{2}$, one can kill the term $x_{1}^{2}$ in $f_{2}$, which gives the desired form (8). After that, one can only act by elements of $T$.

Remark 2. The same family of quartic surfaces is considered in [2, Theorem 2.2], where the authors prove in another way that the lattice of algebraic cycles of the desingularization of a generic surface of this family contains $S_{0}$.

\section{INVARIANTS}

The forms (8) with arbitrary $A, B, C$ constitute a 7 -dimensional $T$-invariant subspace of $Q$. Denote it by $R$. It consists of the forms

$$
F=A x_{0}^{2} x_{2} x_{3}+B x_{1}^{3} x_{3}+C x_{2}^{4}+D x_{1} x_{2}^{2} x_{3}+E x_{2}^{3} x_{3}+G x_{1} x_{2} x_{3}^{2}+H x_{2}^{2} x_{3}^{2} .
$$

It is easy to find the algebra $\mathbb{C}[R]^{T}$ of $T$-invariant polynomial functions on $R$. Fortunately, it turns out to be free.

Proposition 3. The algebra $\mathbb{C}[R]^{T}$ is freely generated by the invariants

$$
I_{4}=A^{2} B D, I_{6}=A^{3} B^{2} E, I_{10}=A^{5} B^{3} C G, I_{12}=A^{6} B^{4} C H
$$

of degrees $4,6,10,12$, respectively.

Proof. Denote by $\alpha, \beta, \gamma$ the weights of the monomials $x_{0}^{2} x_{2} x_{3}, x_{1}^{3} x_{3}, x_{2}^{4}$ with respect to $T$. They are linearly independent, and the weights of the other four monomials of $R$ are $-2 \alpha-\beta,-3 \alpha-2 \beta,-5 \alpha-3 \beta-\gamma,-6 \alpha-4 \beta-\gamma$, whence the result follows.

The next and more difficult step is to understand the relation between the algebra $\mathbb{C}[R]^{T}$ and the algebra $\mathbb{C}[Q]^{\mathrm{SL}_{4}}$ of $\mathrm{SL}_{4}$-invariant polynomial functions on $Q$.

The restriction of invariants defines a homomorphism

$$
\mu: \mathbb{C}[Q]^{\mathrm{SL}_{4}} \rightarrow \mathbb{C}[R]^{T}
$$

and, thereby, a morphism

$$
\mu^{*}: R / / T \rightarrow Q / / \mathrm{SL}_{4}
$$

of the categorical quotients.

Proposition 4. The morphism $\mu^{*}$ is finite.

Proof. This is a particular case of [14, Proposition 11]. (Our space $R$ plays the role of the space denoted by $R_{0}$ in that proposition.) 
The image of $\mu^{*}$ is $\overline{\left(\mathrm{SL}_{4}\right) R} / / \mathrm{SL}_{4}$. It follows from Propositions 2 and 4 that $R / / T$ is its normalization. Since

$$
\operatorname{dim} R / / T=4=\operatorname{dim} \mathcal{L}_{3},
$$

the set of forms $F$ for which $X(F)$ admits a multipolarization of type $\left(h_{0}, S_{0}\right)$ is dense (and open) in $\overline{\left(\mathrm{SL}_{4}\right) R}$.

\section{Singularities}

It is known (see, e.g., [14, Proposition 9]) that the forms of $Q^{\circ}$ are stable in the sense of Mumford [8]. It follows that the image of $Q^{\circ}$ under the quotient morphism $\pi: Q \rightarrow Q / / \mathrm{SL}_{4}$ is open and the restriction of $\pi$ to $Q^{\circ}$ is a geometric quotient as a morphism onto its image; see, e.g., [12, Theorem 4.2]. For this reason, we denote this image as $Q^{\circ} / \mathrm{SL}_{4}$.

Following the lines of [14, we will now prove that the intersection $R^{\circ}=: R \cap Q^{\circ}$ is large enough in a sense.

Proposition 5. The complement of $R^{\circ} / T$ in $R / / T$ is of codimension $\geq 2$.

Proof. Let $R_{1} \subset R$ be the 5 -dimensional subspace defined by the equations $D=E=0$. Clearly, its image $R_{1} / / T \subset R / / T$ is the 2-dimensional subspace defined by the equations $I_{4}=I_{6}=0$.

It follows from [14, Proposition 13] (which is a slightly more general result) that any form

$$
F=A x_{0}^{2} x_{2} x_{3}+B x_{1}^{3} x_{3}+C x_{2}^{4}+G x_{1} x_{2} x_{3}^{2}+H x_{2}^{2} x_{3}^{2} \in R_{1}
$$

with $A, B, C \neq 0$ and $G \neq 0$ or $H \neq 0$ belongs to $Q^{\circ}$. Note that all the forms $F \in R_{1}$ that do not satisfy this condition go to 0 under the quotient morphism $R \rightarrow R / / T$. Thus, $R^{\circ} / T \supset R_{1} / / T \backslash\{0\}$. This implies that the complement of $R^{\circ} / T$ in $R / / T$ cannot contain divisors, since any such divisor would intersect the subspace $R_{1} / / T$ along a line.

\section{MAIN THEOREM}

Theorem 1. The algebra of even Siegel modular forms of genus 2 is freely generated by forms of weights 4, 6, 10, 12 .

Proof. As was explained in Section 2, the algebra of even Siegel modular forms of genus 2 is isomorphic (as a graded algebra) to the algebra $A\left(\mathcal{D}_{3}, \Gamma\right)$ of automorphic forms on the symmetric domain $\mathcal{D}_{3}$ of type IV with respect to the group $\Gamma$ defined by (2). As in [14. Subsection 4.4], it follows from Propositions 2, 4, 5 that this algebra is isomorphic to $\mathbb{C}[R]^{T}$. The structure of the latter algebra is described by Proposition 3 , whence the result follows.

\section{JACOBIAN}

Let $f_{4}, f_{6}, f_{10}, f_{12}$ be free generators of degrees $4,6,10,12$ of the algebra $A\left(\mathcal{D}_{3}, \Gamma\right)$. We will consider them as holomorphic functions on

$$
\mathcal{L}_{3} \subset \mathbb{C}^{2,3}=: T_{0} \otimes \mathbb{C} .
$$

Let $f_{2}$ be the scalar square on $\mathbb{C}^{2,3}$, and let $z_{1}, z_{2}, z_{3}, z_{4}, z_{5}$ be the coordinates on $\mathbb{C}^{2,3}$ with respect to some basis.

Although the functions $f_{4}, f_{6}, f_{10}, f_{12}$ are defined only on (an open subset of) the hypersurface $f_{2}=0$, one can consistently define the Jacobian

$$
J=\frac{D\left(f_{2}, f_{4}, f_{6}, f_{10}, f_{12}\right)}{D\left(z_{1}, z_{2}, z_{3}, z_{4}, z_{5}\right)}
$$


as a holomorphic function on $\mathcal{L}_{3}$. Indeed, the differentials of $f_{4}, f_{6}, f_{10}, f_{12}$ at some point of $\mathcal{L}_{3}$ are linear forms on the tangent space of the hypersurface $f_{2}=0$. In order to define the Jacobian, these forms should be extended to linear forms on the whole space $\mathbb{C}^{2,3}$. These extensions are not unique, but they are defined up to addition of some multiples of $d f_{2}$, which does not affect the Jacobian.

Up to a scalar multiple, the Jacobian $J$ does not depend on the choice of free generators of the algebra $A\left(\mathcal{D}_{3}, \Gamma\right)$. It is homogeneous of degree

$$
2-4-6-10-12-5=-35
$$

and skew-invariant under the group $\Gamma$ in the following sense:

$$
\gamma J=(\operatorname{det} \gamma) J \quad \text { for } \quad \gamma \in \Gamma \text {. }
$$

Thus, $J$ is an automorphic form of weight 35 for the subgroup $\Gamma_{0}$ of $\Gamma$, and $J^{2}$ is an automorphic form of weight 70 for the very group $\Gamma$.

Proposition 6. The null divisor of $J$ is the sum of all the mirrors of (the reflections of) the group $\Gamma$ taken with multiplicity 1.

Proof. Since the quotient map $\pi: \mathcal{L}_{3} \rightarrow \mathcal{L}_{3} / \Gamma$ locally at a point $z \in \mathcal{L}_{3}$ looks like the quotient by the stabilizer $\Gamma_{z}$ of $z$ in $\Gamma$, the Jacobian $J$ vanishes at $z$ if and only if $\Gamma_{z} \neq\{e\}$. Since $\mathcal{L}_{3} / \Gamma$ is a complex manifold (an open subset in $\mathbb{C}^{4}$ ), each stabilizer is generated by reflections. It follows that $J$ vanishes exactly on the mirrors of $\Gamma$. If $z$ belongs to just one mirror, the group $\Gamma_{z}$ is generated by one reflection. It follows that the multiplicity of each mirror in the null divisor of $J$ is 1 .

Theorem 2. The algebra $A\left(\mathcal{D}_{3}, \Gamma_{0}\right)$ is generated by the forms $f_{4}, f_{6}, f_{10}, f_{12}$, and $J$, with the defining relation

$$
J^{2}=P\left(f_{4}, f_{6}, f_{10}, f_{12}\right),
$$

where $P$ is some polynomial.

Proof. Any automorphic form $f$ for $\Gamma_{0}$ decomposes as $f=f_{+}+f_{-}$, where $f_{+}$(resp. $f_{-}$) is a $\Gamma$-invariant (resp. $\Gamma$-skew-invariant) homogeneous holomorphic function of the same degree. The function $f_{+}$is an automorphic form for $\Gamma$, while $f_{-}$vanishes on all the mirrors of $\Gamma$ and, hence, is divisible by $J$, the quotient being an automorphic form for $\Gamma$. Thus, as a vector space, the algebra $A\left(\mathcal{D}_{3}, \Gamma_{0}\right)$ decomposes as

$$
A\left(\mathcal{D}_{3}, \Gamma_{0}\right)=A\left(\mathcal{D}_{3}, \Gamma\right) \oplus A\left(\mathcal{D}_{3}, \Gamma\right) J
$$

whence the result follows.

Corollary. The algebra of all Siegel modular forms of genus 2 is generated by forms $\Phi_{4}, \Phi_{6}, \Phi_{10}, \Phi_{12}, \Phi_{35}$ of weights $4,6,10,12,35$, with the defining relation

$$
\Phi_{35}^{2}=P\left(\Phi_{4}, \Phi_{6}, \Phi_{10}, \Phi_{12}\right) .
$$

The polynomial $P$ is written in [7, p. 849].

The roots of the lattice $T_{0}$ are the vectors $e \in T_{0}$ with $(e, e)=-2$. One can prove that they decompose into two $\Gamma$-orbits depending on whether or not $e \in 2 T_{0}^{*}$. This means that there are two conjugacy classes of reflections in $\Gamma$, say $C_{1}$ and $C_{2}$. The quotient map $\pi: \mathcal{L}_{3} \rightarrow \mathcal{L}_{3} / \Gamma$ takes the mirrors of these two classes of reflections to two irreducible components of the discriminant of $\pi$ given by the equation $P=0$ in $\mathcal{L}_{3} / \Gamma \subset \mathbb{C}^{4}$. It follows that the polynomial $P$ decomposes into a product of two irreducible polynomials, and, correspondingly, the Jacobian $J$ decomposes into a product of two holomorphic functions, say $J_{1}$ and $J_{2}$, so that $J_{1}$ (resp. $J_{2}$ ) vanishes (with multiplicity 1 ) exactly on the mirrors of the reflections of $C_{1}$ (resp. $C_{2}$ ). These functions are automorphic forms for some index 2 subgroups of $\Gamma$. 
Let $e \in T_{0}$ be a root of the class $C_{1}$, i.e. $e \in 2 T_{0}^{*}$. Then $T_{0}=\mathbb{Z} e \oplus 2 U$. As in [14, Section 3], one can prove that the morphism

$$
\nu: \mathcal{L}_{2} / \mathrm{O}^{+}(2 U) \rightarrow \mathcal{L}_{3} / \Gamma
$$

induced by the embedding $2 U \subset T_{0}$, is a closed embedding. This means that the restriction of automorphic forms defines a surjective homomorphism

$$
\rho: A\left(\mathcal{D}_{3}, \Gamma\right) \rightarrow A\left(\mathcal{D}_{2}, \mathrm{O}^{+}(2 U)\right)
$$

Taking into account that $\mathcal{D}_{2}$ is the direct product of two copies of the upper half-plane and $\mathrm{O}^{+}(2 U)$ is an index 2 extension of the direct product of two copies of the Klein modular group, it is easy to prove that the algebra $A\left(\mathcal{D}_{2}, \mathrm{O}^{+}(2 U)\right)$ is freely generated by forms of weights $4,6,12$. It follows that the kernel of $\rho$ is the principal ideal generated by $f_{10}$. This means that $J_{1}^{2}=f_{10}$, which agrees with the explicit form of the polynomial $P$ given in [7].

\section{REFERENCES}

[1] N. D. Allan. On the commensurability class of the Siegel modular group, Bull. Amer. Math. Soc. 74 (1968), 115-118. MR0220674 (36:3726)

[2] A. Clinghera, C. F. Doran. Lattice polarized K3 surfaces and Siegel modular forms, Adv. Math. 231 (2012), 172-212. MR2935386

[3] I. V. Dolgachev. Reflection groups in algebraic geometry, Bull. Amer. Math. Soc. 45 (2007), 1-60. MR:2358376 (2009h:14001)

[4] F. Galluzzi, G. Lombardo (with an Appendix by I. Dolgachev). Correspondences between K3 Surfaces, Michigan Math. J. 52 (2004), 267-277. MR2069800(2005j:14054)

[5] J. Igusa. On Siegel modular forms of genus two, Amer. J. Math. 84 (1962), 175-200. MR.0141643 $(25: 5040)$

[6] J. Igusa. On Siegel modular forms of genus two. II, Amer. J. Math. 86 (1964), 392-412. MR.0168805 (29:6061)

[7] J. Igusa. Modular forms and projective invariants, Amer. J. Math. 89 (1967), 817-855. MR0229643 $(37: 5217)$

[8] D. Mumford. Geometric Invariant Theory, Berlin: Springer, 1965. MR0214602 (35:5451)

[9] V. V. Nikulin. Quotient-groups of groups of automorphisms of hyperbolic forms by subgroups generated by 2-reflections. Algebro-geometric applications, J. Soviet Math. 22 (1983), 1401-1476.

[10] M. Oura. Invariant theoretical approaches to the ring of Siegel modular forms and the related topics (a survey), The 4-th Spring Conference on Modular Forms and Related Topics "Siegel Modular Forms and Abelian Varieties", Hamana Lake, Japan, February 5-9, 2007 (slides).

[11] I. I. Piatetski-Shapiro, I. R. Shafarevich. A Torelli theorem for algebraic surfaces of type K3. Math. USSR: Izvestija 5 (1971), 547-587.

[12] V. L. Popov, E. B. Vinberg. Invariant theory. Encyclopaedia of Mathematical Sciences, Vol. 55, Algebraic Geometry IV, Springer-Verlag, 1994. MR1309681 (95g:14002)

[13] E. B. Vinberg. On the unit groups of some quadratic forms. Math. USSR, Sbornik 16 (1972), 17-35.

[14] E. B. Vinberg. Some free algebras of automorphic forms on symmetric domains of type IV. Transformation Groups 15, N3 (2010), 701-741. MR2718942(2011k:32033)

[15] E. B. Vinberg. On the algebra of Siegel modular forms of genus 2. Preprint 12-088, SFB 701, Universität Bielefeld, 2012, 14 pp.

Department of Mechanics and Mathematics, Moscow State University, Moscow 119992, GSP-2, RUSSIA 